\title{
Evaluation of Functional Foods by Network Methods
}

\author{
Hongmeng $\mathrm{Mu}^{*}$
}

Railway Police College, China

\begin{abstract}
Functional foods are part of the continuum of products that individuals may consume to improve their health and/or contribute to reducing their disease burden. By these networks, various parameters can be calculated to quantitatively characterize the complex and nonlinear physiological effects of wireless communication network and the metabolic networks formed around the central metabolic pathways in vivo. It is concluded that network methods, particularly the metabolic network and intercellular wireless network methods play an important role in quantitative evaluation of functional foods in vivo using only a few milliliters of peripheral blood from volunteers.
\end{abstract}

Keywords: Directed and weighted networks, functional foods, intercellular wireless communication network, metabolic network, quantitative evaluation.

\section{INTRODUCTION}

A functional food is a food provided for an additional function (often one related to health-promotion or disease prevention) by adding new ingredients or more of existing ingredients. The general category of functional foods includes processed food or foods fortified with healthpromoting additives, like "vitamin-enriched" products. Products considered functional generally do not include products where fortification is carried out to meet government regulations and the change is not recorded on the label as a significant addition ("invisible fortification"). An example of this type of fortification can be the historic addition of iodine to table salt, or Vitamin D to milk, performed to resolve public health problems such as rickets. Fermented foods with live cultures are considered as functional foods with probiotic benefits. The term was first used in Japan in the 1980s where there was a government approval process for functional foods called Foods for Specified Health Use (FOSHU). Although many argumentations exist regarding functional foods, several essential problems are still confronted in the course of functional study of functional foods.

At first, although under nutrition relatively causes nutrition deficiency, over nutrition is also the cause of many modern diseases such as obesity, hypertension, hyperlipidemia, diabetes and even cancers. Secondly, according to numerous recent studies, it is indicated that body's nutrition seems to guarantee the function of immune defense system in the first place. Then how the effects of functional foods would be quantified? Though, a number of effects of immune adjustment have been accumulated such as the adjustment to cell factors, but there are no quantitative ways to evaluate the complex immune system. Thirdly, the prominent problem in experimental means is that the evaluation of foods' function is always carried out by means of evaluating

*Address correspondence to these authors at the Railway police college, China; Tel: +358-6-3247476; Fax: +358-6-3247457; E-mail: hunter2011@foxmail.com drug experiments and efficacy in pharmacy and medicine fields. Fourthly, foods can be absorbed only by gastrointestinal organs, otherwise they are excluded. Thus, the question arises of how do foods biologically function on body's organs or tissues? This paper is based on the development of relative studies conducted locally and overseas, providing a synthesis and solution to these problems [3, 4].

The functional evaluation of food has long been controversial, mainly because it is not confirmed that whether the results from animal experiments and methods in vitro exactly represent the functions in human body. Even among human individuals, there are obvious differences, so the results of animals' experiments certainly cannot provide valuable references for humans. The reason for the limited availability of human subjects for functional evaluation of foods mainly lies in the fact that the existing evaluation methods for functional foods are mainly derived from medicinal practices and pathogenic animal models are often required to evaluate the functionality and efficacy of oral medicinal products, despite health being the main concern rather than therapeutic purposes of foods. Another key problem is how to quantitatively evaluate the physiological changes in vivo for the functions of functional foods. In this paper, a review about these areas is carried out, and the network methods are emphasized for their important role in quantitative evaluation of complex and nonlinear problems. The wireless cell-cell communication networks and metabolic networks which belong to the directed and weighted networks are reviewed as well as flux control analysis is carried out. Bio macromolecule interaction networks as the expanded forms of the undirected and unweight networks are currently a major research area.

\section{FUNCTIONAL FOODS AND ITS MAIN MECHA- NISM OF FUNCTION}

Since the prevalence and spread of modern civilization diseases in recent years, many scientists, especially in cytology and molecular biology fields, have developed a huge interest and have acquired world-renowned 
achievements by carrying out lots of researches on foods, nutrition and healthy diet, especially on foods' function on micro ecology in gastrointestinal system, the signal pathway of nutrition absorption and control, the receptors of nonnutrients--phytochemicals and the signal transmission, gastrointestinal functions of neuroendocrine system 10], and gastrointestinal mucous membrane immune and its mechanism of function.

\subsection{The Signal Transmission in Gastrointestinal System and Neuro Conduction Function}

According to the numerous research results, gastrointestinal system can be bound up tightly with the central nerve of cranial nerve by vegetative nerve, and many neuro diseases are closely related to foods. The mechanism by which brain controls eating through appetite has accumulated many research results [4] and has shown that brain has the sensing of foods and nutrition. Except for the color that is transmitted by optical signals; the senses of smell, taste and satiety are transmitted by the mutual recognition of smell elements, taste elements and nutritional or no nutritional elements between the receptor and foods Fig. (1).

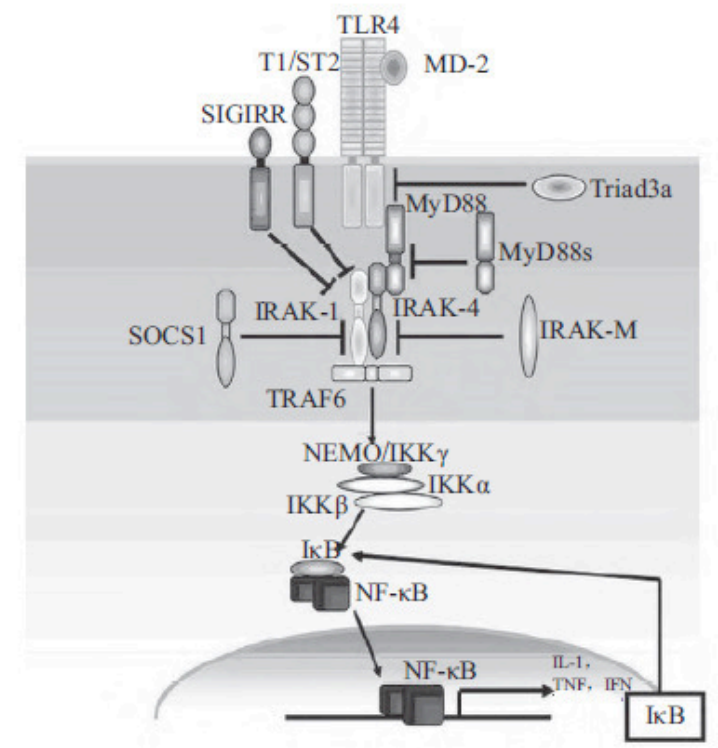

Fig. (1). The balance between inflammatory and anti-inflammatory reactions.

Through the amplification cascade of weak interaction signals in cells, the calcium channel and the vitality of sodium potassium pump change, hence the quantitative amount of electrochemical signals released by sense cells stimulates nearby nerve endings and transmits the complex senses of smell, taste, nutritional and non- nutritional signals to the brain, and in turn controls the appetite and eating process [7].

\subsection{Nutrition Absorption and its Mechanism of Adjustment}

In the recent decades, there have been many IECs receptors and carrier mediators transmitting those signals which have been gained and identified. It also has been proven that the nutritional transmission occurs not only in tongue and nasopharynx but also in the whole gastrointestinal system [5]. It seems that stomach and intestinal channels can pass the characteristics and concentrations of chimes' of different components by the complex and elaborate receptor system. The receptors distribute along the gastrointestinal channels, responding to the consumed foods quantitatively. Although many GPCRs participate in the absorption [JEONG $\mathrm{H}$, TOMBOR B, ALBERT R, et al 2000] of and examination of foods in gastrointestinal systems, the sole nutritional transmission activates multi-crossed signal pathways. Besides, a cell may have many receptors, activating different signal pathways inside the cell. Therefore, the body may activate structural domain inside sense cells by these complex weak interactions between the receptors and ligands, and register the quantitation of output signals by the "communication" of signal pathways.

\subsection{Non- Nutritional Components' Effective Pathways}

Recently, the protection function of phytochemicals in foods has acquired much attention by the academic community. It has long been noticed that though these compounds from plants such as fruits and vegetables are not the classic sense of "big nutrition" like sugar, fat and amino acid, their nutritional effects cannot be neglected. Also, it has been proven that in health protection, anti-cancer, cancer prevention, and especially modern civilization disease prevention show great potential applications. Studies have found that they pass signals to the organism, tissues and cells primarily through interaction and receptors. The major receptors of phytochemicals are Toll-like receptors (TLRs), GPCRs and estrogen receptors (ERs), in which estrogen receptors are nuclear receptors, including lipid-regulating peroxisome proliferator activated receptors (PPARs), the liver $\mathrm{X}$ receptors and farnesoid $\mathrm{X}$ receptors and the pregnane nuclear receptors (PXR); the major signal pathways are phosphoinositide 3 kinase (PI3K), mitogen activated protein kinase (MAPK) and protein kinase $\mathrm{C}$ (PKC); the major transcription factors involved are Nrf2, activator protein-1 (AP-1), nuclear factor $\kappa \mathrm{B}(\mathrm{NF}-\mathrm{\kappa B})$, etc. The same signaling pathway "talks" to each other and thus constitutes the very complex signal transduction network. Body complex life activities, especially the immune system and defense activity depending on the temperature rise and strengthened metabolism, especially the central metabolic pathways involved in the enzyme activity of enhanced coordination, provided energy to cells and body. At the same time, inevitably leading to damage itself, which causes overstressed immune response, is also animportant cause of modern civilization diseases. It is important to note that almost all phytochemicals (PhCs) show inhibition of inflammation, limit innate immunity and acquired immunity, catabolism and endocrine. (AUTHOR: Please review the highlighted vague sentence). Visibly, safeguarding human health today is to limit, rather than enhance immunity.

\subsection{The Intestinal Microecology of Complex Function and Means to Regulate Body's Health}

In recent years, the gut microbes, prebiotics and micro ecological studies have made remarkable achievements. It has been proven that the compositions of intestinal flora in 
the host play an important role in health and life activities. In 2012, Science magazine published a series of summaries about the gut microbes and health relationships fom ecology, genomics, metabolomics, immunology and public health. It relates to the associations between gut microbes and health and disease from multi angles. Costello emphasized that the gut microbes are present in a variety and diversity in its interactions with intestinal mucous membrane system for a clearer understanding of the kinetics of intestinal symbiotic and ecological theory and the eating habits and micro ecological change and stability of the relationship. Lemon focused on the antibiotic treatment caused by ecological damage, and about how to repair the problem by oligo. Nicholson stated that gut microbes are also the participants of host metabolic system which are influenced by dietary nutrition and in turn affect body's metabolic network. Haiser concluded that gut microbes on interaction with medicine and food influence the metabolism. Hooper focused on the relationship between gut microbes and host immune, and guts microbes to maintain a balance of intestinal function. No matter from which side, interactions between the intestinal microbial flora and the host in metabolism, physiology, balance and immune are complex and nonlinear. For example, it is possible that diet structure shapes gut microbes, or gut microbes in turn shape hosts' microecologyas the diet structure is an obvious important medium of gut microbes, while metabolism groups formed by gut microbes and host together play an important role in nutrition absorption and metabolic balance. The fact that needs to be emphasized is that the function of the host' immune system is indispensable to intestinal microbial flora, because the sponsor of the inflammatory response is the host instead of gut microbes. Unfortunately, for such a complex system, in addition to the genetic and inherited diversity of microbial populations, yet there is not a quantitative method to describe complex interactions of the microbes and hosts in metabolic, immune and physiological, endocrine (AUTHOR: The highlighted is vague. Pleasere-phrase to clarify). The reason is that in the face of such a complex nonlinear system, we seem to be helpless. However, if analyzed in the opposite way, it can be found that no matter how much complex the interactions are between foods and host in the gut microbes, they need the host' nerve, metabolism, immunity and endocrine to play a role. Therefore, as long as the quantitative model of wireless network in the body cells on foods result is established, the problem can be solved.

\section{THE COMPLICATION OF NEUROENDOCRINE PHYSIOLOGICAL METABOLISM AND NON- LINEAR ISSUE}

More and more research results demonstrate that foods, functional foods in particular, have obvious influence on neuroendocrine and physiological metabolism Fig. (2). Moreover, the maladjustment of immunity, chronic inflammation, diabetes, hyperlipidemia, obesity, etc. in lifestyles is also explained [1].

Diseases such as anorexia, overeating, depression, mania and loneliness are also seen as the cause of improper diets and imbalance of appetite control. Chronic colitis, Crohn's disease and dementia are closely related to foods. The already conducted researches and studies show that the

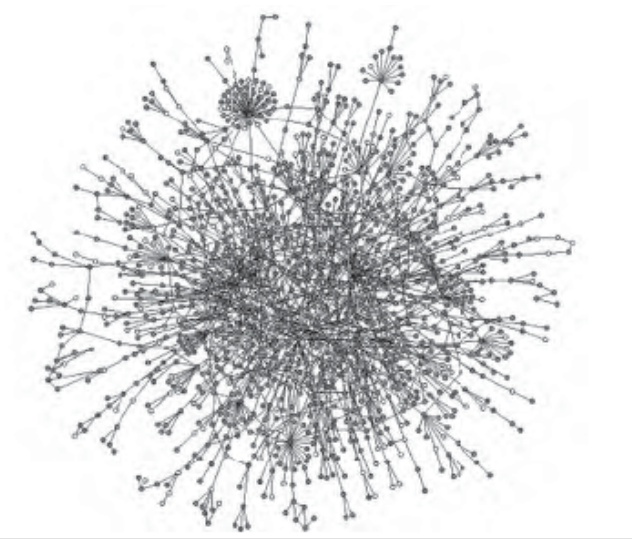

Fig. (2). Yeast protein-protein interaction network.

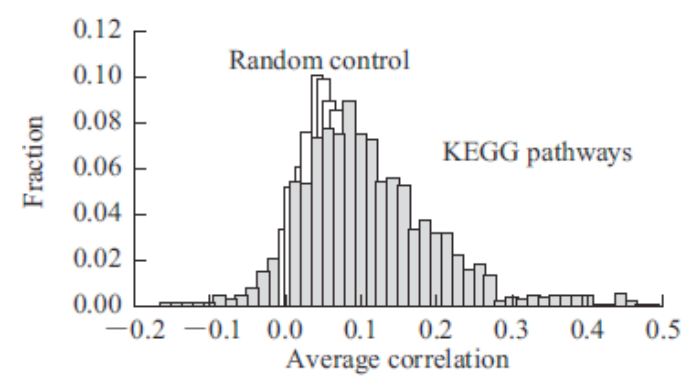

Fig. (3). Average correlation.

above-mentioned diseases occur due to cytokine abnormality, hormone and endocrine disorders. However, because of the presence of non-linear system in function process, that is, molecular mechanism in the complexion of metabolism and interactions, it is hard to establish the quantitative research method and mathematical model as shown in Fig. (3).

\section{INTERNET AND NETWORK METHODS SUD- DENLY RISE IN BIOLOGICAL FIELDS}

The application of network research methods has aroused a revolution in life science fields. The interactive network of Bio macromolecular (DNA, RNA, Protein such as transcription factor) has changed many life science fields, especially the aspects of bio molecular fields. Although many researches and summary papers have been published in many top magazines such as Nature, Science and Cell, and the interactive network of many model creatures, saccharomyces in particular, have been studied extensivelyand subversive conclusions have been drawn, the basic element of life activities is cell rather than the macromolecule. All the bioactive molecules h play biological roles by cells. It is a pity that although scientists have already known the existence of the brain-central nerve system inside the higher animals which controls body's physical and nerve activity as well as the wireless communicative network commanding metabolism, endocrine and immune defense system, until now those researches are generally on a verbal level only.

According to numerous metabolism research results in animal, plant and microbiology, cell's metabolism network has been drawn. But the network is just the estimation of 


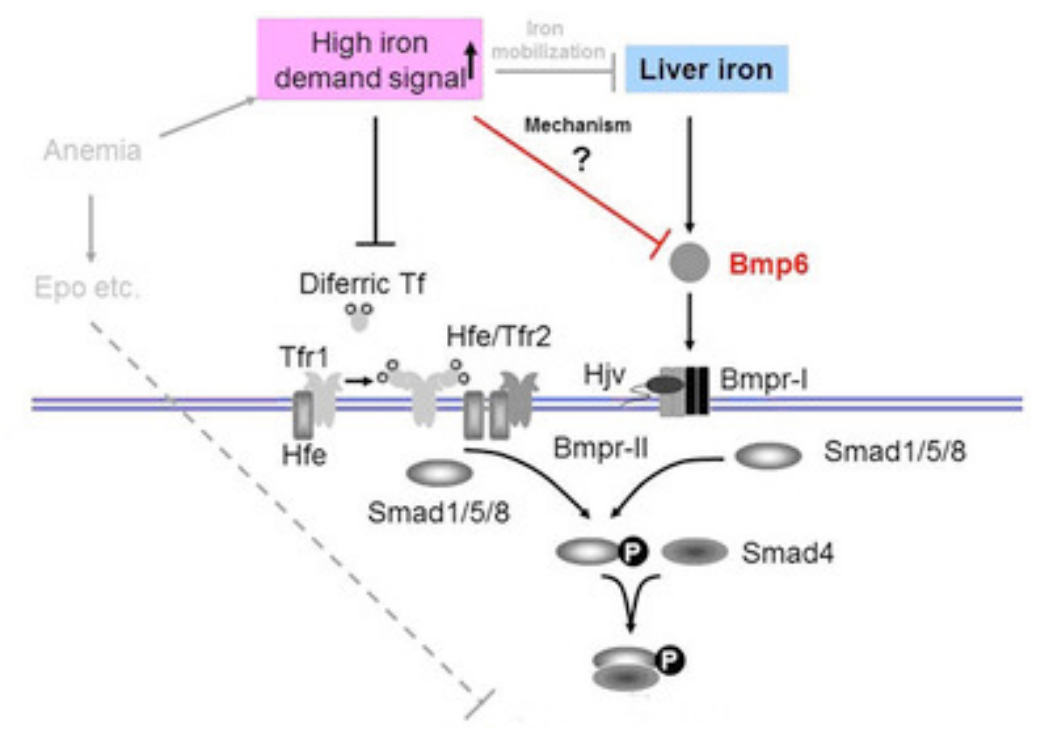

Fig. (4). The hierarchical module of the metabolic network.

chemical structure, enzyme, chemical balance and free energy rather than the real situation inside body and cell. With the development of research, it has been found that through model microbiology especially in Escherichia coli, the fluxcontrol analysis of metabolism network is not what biochemists predict that rate-limiting step and enzyme exist . In fact, it implicates that many biochemical and molecular biology predictions made, do not exist. With the development and application of high throughput measurement techniques like gene manipulation, gene chip, nuclear magnetic resonance, etc., the research of metabolism network has achieved worldrenowned success. In network theory, the interactions between proteins in cells, metabolism, signals and transcription contribute to the formation of the network, with the interactions forming the network of networks. The network of networks is responsible for the activity of body, organs, tissues and cells.

Functional Food is a natural or processed food that contains known biologically-active compounds which when in defined quantitative and qualitative amounts, provides a clinically proven and documented health benefit, and thus, is an important source in the prevention, management and treatment of chronic diseases of the modern age". This was debated at the 9th International Conference on "Functional Foods and Chronic Diseases: Science and Practice" at the University of Nevada, Las Vegas on March 15-17, 2011. Functional Food Center has adopted a new definition of functional food. Functional foods are an emerging field in food science due to their increasing popularity with healthconscious consumers and the ability of marketers to create new interest in existing products. Some countries, such as Canada, Sweden, the United States and the European Union, have specific laws concerning the labelling of such products. In the United States, the kinds of claims which are allowed are overseen and regulated by the Food and Drug Administration (FDA). However, some claims fall outside the range of the FDA and are accompanied by the disclaimer: "These statements have not been evaluated by the Food and Drug Administration. This product is not intended to diag- nose, treat, cure, or prevent any disease." The development of brain nerve network and internet has not only changed the whole science but also the lifestyles of the people and even the thought patterns. Network methods have been widely used in the study of molecular interactions of DNA, RNA and protein, changing front-line scientists' recognition and thinking ways and arousing revolution in molecular biology.

In the metabolism fields, through the research of metabolism network, the complexity and diversity of variants in metabolism groups can be dramatically reduced. The functional evaluation of functional foods is full of challenges. The writer has initially established an evaluation method fit for functional evaluation of foods: wireless communicative network inside cells. Since foods and nutrition are closely related to each other, the writer presents the analysis of flux control method that can study the compound, catabolism and food's function quantitatively inside the body.

Despite nutritional advantages, the functional quality of cereal foods tends to decline when fortified with legumes. Traditional African cowpea-fortified sorghum foods were evaluated using instrumental and descriptive sensory analyses. Fortification reduced uji (fermented thin porridge) pasting peak viscosity and cool paste viscosity by up to $23 \%$ and $12 \%$, respectively, probably as a result of starch content being reduced. NS 5511 injera (fermented thin flatbread) stiffness increased by up to $25 \%$, probably due to a reduction in tannin content. Orbit injera stiffness reduced the descriptive sensory panel by up to $22 \%$. There was a $20 \%$ decrease in instrumental firmness of NS 5511 ugali as a result of fortification, which was not detected by the sensory panel. Fortification had generally no effect on the stickiness of ugali. Fortified ugali had a characteristic cowpea flavour. Principal component analysis (PCA) of ugali sensory attributes indicated a $30 \%$ variation due to the presence of cowpea. Most of the variation $(47 \%)$ was attributed to sorghum cultivar. Sensory panel analysis concerning ugali firmness and stickiness correlated positively with the instrumental texture analysis. Fortification with cowpea affects texture and taste of traditional African sorghum foods, the extent of which large- 
ly depends on the sorghum cultivar concerned. Instrumental texture analysis relates well with human perception of sorghum food texture and can rapidly predict the effects of fortification with legumes on such foods.

Recently, a semi-automated yeast two-hybrid screens technique and SPR technique about micro-molecular interactions have been able to let scientists measure when and how those micro molecules interact with each other. These studies have revealed intracellular molecular interaction network' architecture characteristics, which are like internet, computer chip and social network. The research about protein-protein interaction network shows that Scale-free network has a remarkable ability to resist accidental injury. Even though $80 \%$ of randomly selected nodes are broken, the remaining $20 \%$ can still form interconnected clusters, maintaining the connection between any two nodes. This reminds us that there are very few single genetic traits, especially facing the complex non-linear system. It is necessary to design the operation scheme from the point of view of system and network, emphasizing on the consideration of the key factors which influence the stability of the system.

\section{CONCLUSION}

The development of brain nerve network and internet has not only changed the whole science but also people's lifestyles and even the thought patterns. Network methods have been widely used in the study of molecular interactions of DNA, RNA and protein, changing front-line scientists' recognition and thinking pathways and have aroused revolution in molecular biology. In the metabolism fields, through the research of metabolism network, the complexity and diversity of variants in metabolism groups can be reduced dramatically. The functional evaluation of functional foods is full of challenges. The writer initially established an evaluation method fit for the functional evaluation of foods: wireless communicative network inside cells. Since foods and nutrition are closely related to each other, the writer presents the analysis of flux control method that can study the compound, catabolism and function of foods quantitatively inside the body. In fact, the intestinal micro ecology system, fruit and vegetable surface ecosystems, animals, plants and microbial ecosystem, arrested and predators and society in a relationship between person and person actually can constitute a weighted network, which may have a bright future in the field of research.

\section{CONFLICT OF INTEREST}

The author confirms that this article content has no conflict of interest.

\section{ACKNOWLEDGEMENTS}

This work is supported by the Key Project of Guangxi Social Sciences, China (No. gxsk201424), the Education Science fund of the Education Department of Guangxi, China (No.2014JGA268), and Guangxi Office for Education Sciences Planning, China (No.2013C108).

\section{REFERENCES}

[1] S. Aditya, and B. Dasgupta, and M. Karpinski, "Algorithmic perspectives of network transitive reduction problems and their applications to synthesis and analysis of biological networks," Biology, vol. 3, pp. 1-21, 2014.

[2] A. L. Barabási, and Z. N. Oltvai, "Network biology: understand the cell's functional organization," Nature Reviews Genetics, vol. 5, pp. 101-113, 2004.

[3] E. K. Costello, K. Stagaman, L. Dethlefsen, et al. "The application of ecological theory toward an understanding of the human microbiome," Science, vol. 336, pp. 1255-1262, 2012.

[4] J. I. Gordon, and T. H. Y. Honor, Gut symbionts redux[J]. Science, 2012, 336: 1251-1253.

[5] T. Ito, T. Chiba, R. Ozawa, M. Yoshida, M. Hattori, and Y. Sakaki, "A comprehensive two-hybrid analysis to explore the yeast protein interactome," In: Proceedings of the National Academy of Sciences, vol. 98, no. 4569-4574, 2001.

[6] H. Jeong, B. Tombor, R. Albert, Z. N. Oltvai, and A. L. Barabási, "The large-scale organization of metabolic networks," Nature, vol. 407, pp. 651-654, 2000.

[7] K. P. Lemon, G. C. Armitage, D. A. Relman, et al. "Microbiotatargeted therapies: an ecological perspective," Science Translational Medicine, vol. 137, pp. 137-145, 2012.

[8] R. O'lone, M. C. Frith, E. K. Karlsson, et al. "Genomic targets of nuclear estrogen receptors," Molecular Endocrinology, vol. 18, no.8, pp.1859-1875, 2004.

[9] F. Reimann, G. Tolhurst, and F. M. Gribblei, "G-Protein-coupled receptors in intestinal chemosensation," Cell Metabolism, vol. 15, no.4, pp. 421-431, 2012.

[10] M. Vidal, M. E. Cusick, and A. L. Barabasi, "Interactome networks and human disease," Cell, vol. 144, pp. 986-998, 2011.

\begin{tabular}{lcc}
\hline Received: June 10, 2015 & Revised: July 29, 2015 & Accepted: August 15, 2015 \\
C) Hongmeng Mu; Licensee Bentham Open. &
\end{tabular}

This is an open access article licensed under the terms of the (https://creativecommons.org/licenses/by/4.0/legalcode), which permits unrestricted, noncommercial use, distribution and reproduction in any medium, provided the work is properly cited. 